\title{
Prevalence and determinants of type 2 diabetes among lean African migrants and non-migrants: the RODAM study
}

Felix P Chilunga ${ }^{1}$, Peter Henneman², Karlijn AC Meeks ${ }^{1,3}$, Erik Beune ${ }^{1}$, Ana Requena-Méndez ${ }^{4}$, Liam Smeeth ${ }^{5}$, Juliet Addo ${ }^{5}$, Silver Bahendeka ${ }^{6}$, Ina Danquah $^{7}$, Matthias B Schulze ${ }^{8}$, Joachim Spranger ${ }^{9}$, Ellis Owusu-Dabo ${ }^{10}$, Kerstin Klipstein-Grobusch ${ }^{11,12}$, Marcel MAM Mannens ${ }^{2}$, Charles Agyemang ${ }^{1}$

${ }^{1}$ Department of Public Health, Amsterdam Public Health Research Institute, Amsterdam UMC, University of Amsterdam, Amsterdam, the Netherlands

${ }^{2}$ Department of Clinical Genetics, Amsterdam UMC, University of

Amsterdam, Amsterdam, the Netherlands

${ }^{3}$ Center for Research on Genomics and Global Health, National Human Genome Research Institute, National Institutes of Health, Bethesda, Maryland, USA

${ }^{4}$ ISGlobal, Barcelona Centre for International Health Research (CRESIB), Hospital Clinic, University of Barcelona, Barcelona, Spain

${ }^{5}$ Department of Non-communicable Disease Epidemiology, London School of Hygiene and Tropical Medicine, London, United Kingdom

${ }^{6}$ Department of Medicine, MKPGMS-Uganda Martyrs University, Kampala, Uganda

${ }^{7}$ Department of Molecular Epidemiology, German Institute of Human Nutrition, Nuthetal, Germany

${ }^{8}$ Institute for Social Medicine, Epidemiology and Health Economics, Berlin Institute of Health, University of Berlin, Berlin, Germany

${ }^{9}$ Clinic of Endocrinology, Diabetes and Metabolism, Berlin Institute of Health, University of Berlin, Berlin, Germany

${ }^{10}$ School of Public Health, Kwame Nkrumah University of Science and Technology, Kumasi, Ghana

${ }^{11}$ Julius Global Health, Julius Centre for Health Sciences and Primary Care, University Medical Centre Utrecht, Utrecht University, Utrecht, the Netherlands

${ }^{12}$ Division of Epidemiology and Biostatistics, School of Public Health, Faculty of Health Sciences, University of the Witwatersrand, Johannesburg, South Africa

\section{Correspondence to:}

Felix P. Chilunga, MD

Amsterdam Public Health Research Institute

Amsterdam UMC - University of Amsterdam

Meibergdreef 9 1105AZ

Amsterdam

The Netherlands

f.p.chilunga@amc.uva.nl
Background Exposure to adverse conditions earlier in life-course can predispose to type 2 diabetes in adulthood, irrespective of body mass index (BMI). However, the burden of type 2 diabetes in lean Africans is not well understood despite higher exposure to adverse early life conditions. Mirroring ongoing epidemiological transition, we assessed the burden and determinants of type 2 diabetes in a homogenous group of lean Ghanaians residing in rural and urban Ghana, and as migrants in Europe.

Methods Baseline data from 2179 RODAM study participants with $\mathrm{BMI}<25 \mathrm{~kg} / \mathrm{m}^{2}(25-70$ years) were analyzed. Prevalence and determinants of type 2 diabetes were estimated using logistic regression analysis. Adjustments were made for socio-demographic and lifestyle factors, use of anti-diabetic medication and optimal blood glucose control.

Results Prevalence of type 2 diabetes in rural, urban and migrant lean participants were $3.5 \%, 8.9 \%$ and $7.5 \%$ respectively, representing $55.4 \%, 35.6 \%, 13.2 \%$ of all participants with type 2 diabetes. Compared with lean rural participants, the odds of type 2 diabetes were higher in lean urban participants (adjusted $\mathrm{OR}=8.81$, $95 \% \mathrm{CI}=6.56-11.06$ ), followed by migrants (5.27, 95\% CI=3.51-6.91). Irrespective of site, determinants of type 2 diabetes in lean participants include; presence of hypertension, physical inactivity, hypercholesterolemia and age ( $>45$ years).

Conclusions Our study shows a high prevalence of type 2 diabetes among lean African populations in different geographical settings. Future studies are needed in-order to examine how contextual differences are related to the pathophysiology of type 2 diabetes in lean individuals. 
It is well established that there is an emerging burden of type 2 diabetes and other cardio-metabolic diseases in sub-Saharan Africa (SSA) [1,2]. Although obesity is a well-known risk factor for type 2 diabetes, populations from low and middle income countries (LMIC) are at increased risk of type 2 diabetes at lower levels of body mass index (BMI) [3]. This is in contrast to high-income countries (HIC) where only about $20 \%$ of individuals with type 2 diabetes have normal BMI $[4,5]$. For many SSA countries, where health care resources are severely constrained, occurrence of type 2 diabetes in underweight/normal individuals poses major public health challenges such as increased mortality as a result of obesity paradox, increased rates of colorectal cancer and inapplicability of universally recommended lifestyle interventions such as body weight reduction [6-8].

Type 2 diabetes in underweight/normal weight individuals has been attributed to exposure to adverse conditions earlier in life-course $[9,10]$. This is in addition to the classical risk factors for type 2 diabetes such as alcohol consumption, smoking and physical inactivity [7]. Despite the higher rates of maternal undernutrition, pre-natal injurious agents and early childhood undernutrition in SSA, the burden of type 2 diabetes in underweight/normal weight Africans is still not well understood [11]. Additionally, there is need for diabetes control strategies to be applied according to specific rural, urban and migrant contexts due to rapid urbanization, migration and epidemiological transition [1]. However, little is also known about the determinants of type 2 diabetes in each of the rural, urban and migrant SSA contexts.

Current reports show that the major pathophysiology of type 2 diabetes in underweight/normal weight individuals is rapid beta cell failure as opposed to insulin resistance [8]. Therefore, investigating the burden of type 2 diabetes in non-obese populations and respective determinants in each geographical context will expand knowledge well beyond the connection between lifestyle factors, insulin resistance and type 2 diabetes. Novel aspects of the relationship between developmental origins of disease and pathophysiology of type 2 diabetes can be unraveled, thereby presenting opportunities for groundbreaking approaches to preventing and managing the disease [12].

In order to accentuate the environmental contributions to development of type 2 diabetes among underweight/normal weight individuals and mitigate the effects of dissimilar genetic background, it is imperative that a homogenous group of people is studied [13]. We, therefore, used data from a homogenous group of Ghanaians who participated in the Research on Obesity and Diabetes among African Migrants (RODAM) study in order to 1 ) assess the prevalence of type 2 diabetes in underweight/normal weight SSA populations in rural, urban and migrant contexts. 2) assess the proportion of types 2 diabetes cases comprised of underweight/normal weight populations in each SSA context. 3) assess the determinants of type 2 diabetes in underweight/normal weight populations in each SSA context. 4) assess the relative contribution of beta cell failure and insulin resistance to type 2 diabetes in underweight/normal weight SSA populations.

\section{METHODS}

\section{Study setting and population}

The multicentre RODAM study was initiated in 2012 with the aim of understanding the complex interplay between the environment and genetics in the development of obesity and diabetes among African migrants. The full details of the study have been published elsewhere [13]. In brief, 5898 Ghanaian men and women aged 25-70 years were recruited in Europe and in Ghana. In Europe, participants were recruited from the cities of Amsterdam (Netherlands), Berlin (Germany) and London (United Kingdom). In Ghana, recruitment of participants in the urban area was conducted in two purposively chosen cities (Kumasi and Obuasi), while recruitment in the rural area was conducted in 15 villages in the Ashanti region. A standardised approach for questionnaires, anthropometric measurements and venepuncture samples was used across all study sites. The response rates were 67\% in Amsterdam, 68\% in Berlin, 75\% in London, $76 \%$ in rural Ghana and 74\% in urban Ghana. Since our study was designed to study international migration, we categorized Ghanaians living in Europe as migrants while Ghanaians living in Ghana were considered as non-migrants.

\section{Ethical approval and consent to participate}

Ethical approval was obtained from ethics committees of involved institutions in Ghana, Netherlands, Germany and UK before the start of data collection. All participants gave written informed consent. 


\section{Underweight/normal weight individuals with Type 2 diabetes}

Presence of type 2 diabetes was defined using the World Health Organization (WHO) diagnostic criteria (fasting blood glucose, $\geq 7.0 \mathrm{mmol} / \mathrm{L}$, or current use of medication prescribed to treat type 2 diabetes or a previous self-reported diagnosis of type 2 diabetes a health professional. We used the current WHO definition of low/normal weight (ie, BMI $<25 \mathrm{~kg} / \mathrm{m}^{2}$ ) to identify underweight/normal weight individuals in our study [14].

\section{Measurements}

The following measurements were obtained through a structured questionnaire; age, sex, educational attainment, history of type 2 diabetes, use of medication for type 2 diabetes, use of dietary treatment for type 2 diabetes, physical activity levels, alcohol consumption, smoking and length of stay in Europe. Education was categorised as follows; (1) none or elementary, 2) lower secondary, 3) higher secondary, and 4) tertiary. Levels of physical activity were calculated using the Global Physical Activity Questionnaire (GPAQ) version 2, and categorised into a low, moderate or high levels based on the GPAQ criteria [15]. Alcohol consumption was categorised into no consumption, or any consumption. Smoking was categorised into current smokers, past smokers or non-smoker. Body weight was measured in light clothing and without shoes with SECA 877 scales to the nearest $0.1 \mathrm{~kg}$. Participants height was measured without shoes with a portable stadiometer (SECA 217) to the nearest $0.1 \mathrm{~cm}$. BMI was calculated as weight (kg) divided by height squared $\left(\mathrm{m}^{2}\right)$. Central obesity was defined as waist-to-hip ratio (WHR) of $\geq 0.95$ for men and $\geq 0.85$ for women. Blood pressure (BP) was measured three times using appropriate cuffs in a sitting position after at least 5 minutes of rest $(\mathrm{mmHg})$. The mean of the last two measurements was used in the analysis.

All biochemical analyses were performed in Berlin with an ABX Pentra 400 chemistry analyser (ABX Pentra; Horiba ABX, Montpellier, France). Concentration of total cholesterol, low density lipoprotein (LDL)-cholesterol, high density lipoprotein (HDL)-cholesterol and triglycerides was assessed using colorimetric test kits (mmol/L). Insulin concentrations were assessed using the Mercodia ELISA kit in pmol/L. Fasting plasma glucose concentration was measured using an enzymatic method (hexokinase) in $\mathrm{mmol} / \mathrm{L}$. Glycated hemoglobin (HbAlc) was measured in \% and mmol/mol (HPLC). Hypercholesterolemia was defined as total cholesterol $\geq 200 \mathrm{mg} / \mathrm{dL}$, low density lipoprotein (LDL) -cholesterol $\geq 160 \mathrm{mg} / \mathrm{dL}$, high density lipoprotein (HDL)-cholesterol $\leq 40 \mathrm{mg} / \mathrm{dL}$, triglycerides $\geq 150 \mathrm{mg} / \mathrm{dL}$ or medication with lipid lowering drugs. Impaired fasting glucose (IFG) was defined as a fasting glucose between 5.6 and $6.9 \mathrm{mmol} / \mathrm{L}$, according to American Diabetes Association. Insulin sensitivity and beta cell function were assessed using Homeostatic Model Assessment (HOMA): HOMA-derived insulin resistance index (HOMA-IR) and HOMA-derived beta cell function (HOMA-B) [16]. HbAlc $<7 \%$ (53 mmol/mol) was used to show optimal blood glucose control in individuals with type 2 diabetes for the preceding two to three months.

\section{Data analysis}

Data analyses were performed using Stata 14 (Stata Corp LP, Texas 77845, USA). Summary statistics were presented as proportions for categorical variables and as means (with standard deviations) for normally distributed continuous variables or medians (with IQR-interquartile range) for skewed continuous variables. The differences of baseline characteristics in rural, urban and migrant participants were tested by Analysis of Variance (ANOVA) for normally distributed continuous variables, Kruskal-Wallis test for skewed continuous variables and $\chi^{2}$ tests for categorical variables. Prevalence of type 2 diabetes was calculated and standardised for age with a direct method. Logistic regression models were used to calculate odds ratios with corresponding 95\% Confidence Intervals (CI) for type 2 diabetes in underweight/ normal weight participants by location of current residency (rural Ghana as the reference category), adjusting for socio-demographic factors. In multivariable models, adjustments were made for alcohol consumption, smoking, physical activity, blood pressure, receiving treatment for type 2 diabetes (weight-loss diet, oral medications and insulin use), level of blood glucose control (HbAlc $<7 \% / 53 \mathrm{mmol} / \mathrm{mol}$ ) and length of stay for migrants. For adjustment variables, missing values represented $<5 \%$ of the data in every variable. In addition, univariate and multivariate logistic regression models were used to assess the associations between type 2 diabetes in underweight/normal weight individuals and its determinants by location of current residency.

In a sub-set of underweight/normal weight participants with IFG, we assessed the association of HOMA-IR and HOMA-B with IFG using logistic regression models. This sub-analysis was carried out in order to identify whether beta cell failure or insulin resistance was more associated with the development of type 
2 diabetes in underweight/normal weight individuals. In these models; first, we calculated the inverse of HOMA-B (ie, 1/HOMA-B). Second, we converted both HOMA-IR and inverse HOMA-B into standardized $z$ scores in order to achieve comparability of the variables. Third, we calculated the odds of IFG by 1SD increase in HOMA-IR and inverse HOMA-B adjusting for age, sex and location of residency. Lastly, we calculated the attributable risk for inverse HOMA-B and HOMA-IR relative to IFG.

\section{RESULTS}

\section{Baseline characteristics}

A total of 2179 (37.1\%) of all RODAM study participants were underweight/normal weight, of which, 853(39.1\%) were rural residents, 583(26.8\%) were urban residents and 743(34.1\%) were migrants (Figure S1 in Online Supplementary Document). In these underweight/normal weight participants, the majority of migrants were male (61.2\%), while the majority of rural and urban participants were females (53.6\% and 55.6\%, respectively). About half (50.1\%) of underweight/normal weight participants were older than 45 years. Median age was highest in rural residents, who were also the least educated. Median length of stay in Europe among migrants was 13 years (IQR, 5.1-22.2). Rural participants were more likely to be former smokers, and had the highest levels of physical activity and triglycerides. Urban participants had the highest level of total cholesterol, LDL-cholesterol, hypercholesterolemia and WHR. Migrants had the highest level of mean BMI, HDL-cholesterol, blood pressure, and were more likely than rural and urban participants to drink alcohol and smoke (Table 1).

\section{Type 2 diabetes in underweight/normal weight individuals}

Median fasting blood glucose and insulin levels were highest in underweight/normal weight urban residents. The crude prevalence of type 2 diabetes in underweight/normal weight participants was 3.6\% among rural residents, $8.8 \%$ among urban residents and $6.2 \%$ among migrants, with an overall prevalence of $5.9 \%$. About $47 \%$ of all underweight/normal weight individuals with type 2 diabetes were on treatment (diet and medication). The most common mode of treatment was oral-antidiabetic agents in all three groups. Use of insulin for type 2 diabetes treatment was more common in migrants. The majority (63\%) of underweight/normal weight migrants on type 2 diabetes treatment had achieved optimal blood glucose control in the preceding 2-3 months (Table 1).

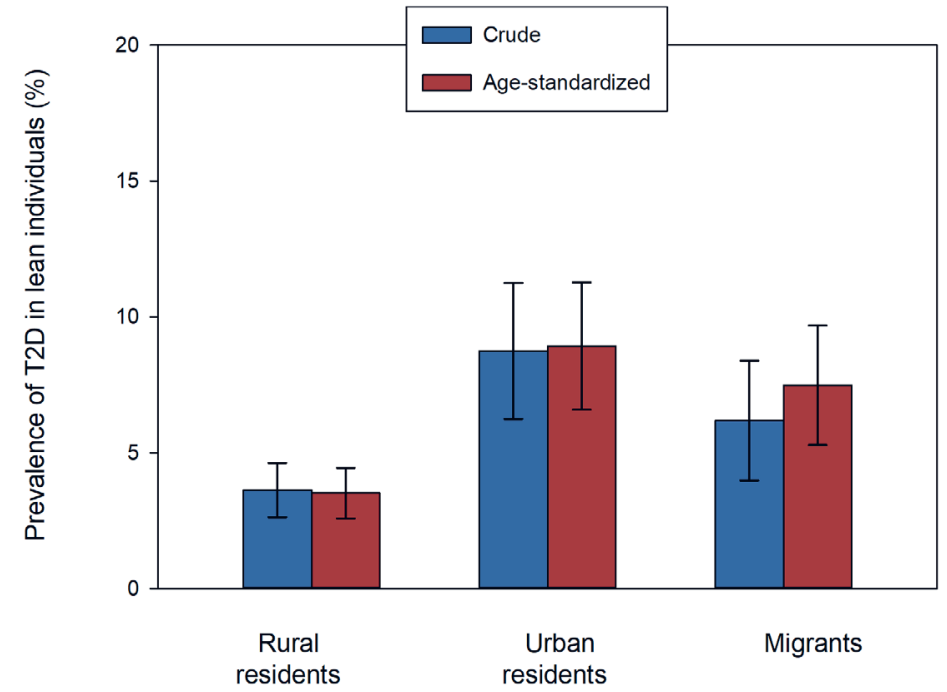

Figure 1. Crude and age -standardized prevalence of type 2 diabetes in underweight/normal weight urban residents, rural residents and migrants. Age-standardized prevalence of type 2 diabetes in underweight/ normal weight participants includes confidence intervals as follows; rural residents 3.52\% (95\% CI $=2.29-4.45)$, urban residents $8.93 \%$ (95\% $\mathrm{CI}=6.59-11.27)$, migrants $7.49 \%(95 \% \mathrm{CI}=4.89-6.85) . \mathrm{T} 2 \mathrm{D}-$ type 2 diabetes mellitus.
When standardized for age, prevalence of type 2 diabetes in underweight/normal weight individuals was $3.5 \%, 8.9 \%$ and $7.5 \%$ in rural residents, urban residents, and migrants, respectively (Figure 1). The proportions of type 2 diabetes in underweight/ normal weight individuals relative to all type 2 diabetes cases were $55.4 \%$ for rural residents, 35.6\% for urban residents and 13.2\% for migrants (Figure 2). Compared to rural residents, the odds of type 2 diabetes in underweight/normal weight individuals were higher in both urban residents $(\mathrm{OR}=8.81$, $95 \% \mathrm{CI}=6.56-11.06)$ and migrants ( $\mathrm{OR}=5.27,95 \%$ $\mathrm{CI}=3.51-6.91)$ compared to rural residents when adjusted for other factors (Figure 3 ).

In the subset of 217 underweight/normal weight individuals with IFG (compared to those with normal blood glucose), the odds for IFG were higher per 1SD increase in inverse HOMA-B $(A O R=16.07$, 95\% CI $=10.16-25.41)$ compared to HOMA-IR ( $\mathrm{AOR}=9.83,95 \% \mathrm{CI}=6.91-13.99)$, with correspondingly higher attributable risk (AR)\% for inverse HOMA-B (AR\% 43.57, 95\% CI=35.30-51.84) than for HOMA-IR (AR\% 32.86, 95\% CI=27.0238.70) (Table S1 in Online Supplementary Document). 
Table 1. Baseline characteristics of participants in migrant, urban and rural residents*

\begin{tabular}{|c|c|c|c|c|}
\hline & TOTAL (n = 2179) & Migrants $(n=743)$ & Urban RESIDENTS ( $N=583$ ) & RuRAL RESIDENTS ( $N=853$ ) \\
\hline \multicolumn{5}{|l|}{ Demographics } \\
\hline Women, n (\%) & $1069(49.1)$ & $288(38.8)$ & $324(55.6)$ & $457(53.6) \dagger$ \\
\hline Mean age, years & 45.07 (13.89) & $41.17(12.74)$ & 44.59 (12.59) & $48.77(14.69) \dagger$ \\
\hline \multicolumn{5}{|l|}{ Age group, $\mathrm{n}(\%)$ : } \\
\hline $25-44$ & $1070(49.11)$ & $429(57.74)$ & $290(49.74)$ & $351(41.15)^{\dagger}$ \\
\hline $45-65$ & $898(41.21)$ & $287(38.63)$ & $257(44.08)$ & $354(41.50)$ \\
\hline$>65$ & $211(9.68)$ & $27(3.63)$ & $36(6.17)$ & $148(17.35)$ \\
\hline \multicolumn{5}{|l|}{ Education, n (\%): } \\
\hline Elementary & $825(40.52)$ & $115(17.04)$ & $239(42.45)$ & $471(59.02)^{\dagger}$ \\
\hline Lower secondary & $700(34.38)$ & $157(34.67)$ & $227(40.32)$ & $239(29.95)$ \\
\hline Higher secondary & $309(15.18)$ & $104(26.81)$ & $66(11.72)$ & $62(7.77)$ \\
\hline Tertiary & $202(9.92)$ & $100(21.48)$ & $31(5.51)$ & $26(3.26)$ \\
\hline \multicolumn{5}{|l|}{ Lifestyle risk factors } \\
\hline \multicolumn{5}{|l|}{ BMI, n (\%): } \\
\hline$<18$ & $206(9.45)$ & $14(1.88)$ & $33(5.66)$ & $159(18.64) \dagger$ \\
\hline $18.0-24.9$ & $1973(90.55)$ & $729(98.12)$ & $550(94.34)$ & $694(81.36)$ \\
\hline Median BMI, kg/m² & $22.01(20.24-23.68)$ & $23.20(21.69-24.16)$ & $22.08(20.64-23.73)$ & $20.58(18.97-22.34) \dagger$ \\
\hline Raised waist-to-hip ratio (WHR), n (\%) & $1074(49.36)$ & $279(37.65)$ & $330(56.70)$ & $465(54.51)$ \\
\hline Mean total cholesterol, mmol/L & $4.67(1.12)$ & $4.85(1.08)$ & $4.94(1.08)$ & $4.35(1.10) \mathrm{c}$ \\
\hline Mean HDL cholesterol, mmol/L & $1.32(0.41)$ & $1.48(0.39)$ & $1.29(0.33)$ & $1.20(0.38) \dagger$ \\
\hline Mean LDL cholesterol, mmol/L & $2.91(0.94)$ & $2.99(0.94)$ & $3.18(0.92)$ & $2.67(0.91) \dagger$ \\
\hline Mean Triglycerides, $\mathrm{mmol} / \mathrm{L}$ & $0.85(0.65-1.14)$ & $0.71(0.56-0.94)$ & $0.89(0.69-1.19)$ & $0.95(0.73-1.23) \dagger$ \\
\hline Hypercholesterolemia, n (\%) & $83(5.94)$ & $13(2.24)$ & $41(11.06)$ & $29(6.79)$ \\
\hline Mean Systolic BP, mmHg & $125.52(19.91)$ & $128.40(17.34)$ & $124.46(21.45)$ & $123.78(20.65) \dagger$ \\
\hline Mean Diastolic BP, mmHg & $78.22(12.04)$ & $80.77(11.63)$ & $77.60(12.59)$ & $76.41(11.63) \dagger$ \\
\hline Hypertension, n (\%) & $609(27.95)$ & $257(34.59)$ & $142(24.36)$ & $210(24.62) \dagger$ \\
\hline High level physical activity, n (\%) & $1023(54.18)$ & $254(47.65)$ & $288(51.43)$ & $481(60.50) \dagger$ \\
\hline \multicolumn{5}{|l|}{ Smoking, n (\%): } \\
\hline Current & $87(4.28)$ & $54(8.00)$ & $10(1.77)$ & $23(2.89)^{\ddagger}$ \\
\hline Past & $156(7.67)$ & $49(7.26)$ & $39(6.91)$ & $68(8.54)^{\dagger}$ \\
\hline Any alcohol consumption, n (\%) & $853(39.15)$ & $320(43.07)$ & $169(28.99)$ & $364(42.67)^{\dagger}$ \\
\hline Median length of stay, years & $13.12(5.11-22.21)$ & $13.12(5.11-22.21)$ & & \\
\hline \multicolumn{5}{|l|}{ Diabetes related } \\
\hline Median fasting blood glucose, $\mathrm{mmol} / \mathrm{L}$ & $4.95(4.60-5.32)$ & $4.91(4.57-5.33)$ & $5.04(4.71-5.40)$ & $4.89(4.55-5.27)^{\dagger}$ \\
\hline Median Insulin levels,(pmol/L) & $3.9(2.5-6.1)$ & $4.3(2.8-6.3)$ & $4.4(2.4-6.8)$ & $3.4(2.2-5.3)^{\dagger}$ \\
\hline Impaired Fasting Glucose, n (\%) & $217(10.58)$ & $80(11.48)$ & $57(10.71)$ & $80(9.73)^{\dagger}$ \\
\hline Mean HOMA-IR & $1.19(1.53)$ & $1.24(1.92)$ & $1.35(1.48)$ & $1.01(1.09)^{\dagger}$ \\
\hline Mean Inverse HOMA-B & $0.03(0.05)$ & $0.02(0.03)$ & $0.03(0.08)$ & $0.02(0.04)^{\dagger}$ \\
\hline Diabetics, n (\%) & $128(5.87)$ & $46(6.19)$ & $51(8.75)$ & $31(3.63)^{\dagger}$ \\
\hline On diabetes treatment, $\mathrm{n}(\%)$ & $60(46.87)$ & $27(58.69)$ & $25(49.02)$ & $8(25.81)^{\ddagger}$ \\
\hline On diabetic diet, $\mathrm{n}(\%)$ & $37(61.67)$ & $11(40.74)$ & $18(72.0)$ & $8(100.0)$ \\
\hline On oral anti-diabetic drugs, n (\%) & $54(90.0)$ & $21(77.78)$ & $25(100.0)$ & $8(100.0)$ \\
\hline On Insulin treatment, n (\%) & $3(5.0)$ & $4(14.81)$ & $3(12.0)$ & 0 \\
\hline HbAlc levels < $7 \%(53 \mathrm{mmol} / \mathrm{mol}), \mathrm{n}(\%)$ & $28(46.67)$ & $17(62.96)$ & $7(28.00)$ & $4(50.00)$ \\
\hline \multicolumn{5}{|c|}{$\begin{array}{l}\text { IQR - interquartile range; SD - standard deviation, BMI - body mass index, HDL - high density lipol } \\
\text { pressure, HOMA - homeostatic model assessment, HOMA-IR - HOMA-derived insulin resistance } \\
\text { function (HOMA-B), HbAlc - glycated hemoglobin } \\
\text { *Data are } \mathrm{n}(\%) \text {, median (interquartile range, IQR), or mean (standard deviation, SD). } \\
\dagger P \text { for difference in baseline characteristics between migrants, urban and rural participants }<0.001 \text {. } \\
¥ P \text { for difference in baseline characteristics between migrants, urban and rural participants }>0.05 \text {. }\end{array}$} \\
\hline
\end{tabular}

\section{Determinants of type 2 diabetes in underweight/normal weight participants}

In underweight/normal weight rural residents, presence of hypertension (AOR $=2.24,95 \% \mathrm{CI}=1.82-6.09$ ) and high levels of physical activity $(\mathrm{AOR}=0.22,95 \% \mathrm{CI}=0.06-0.78)$ were independent determinants of type 2 diabetes. In underweight/normal weight urban residents, middle age (45-65 years) ( $\mathrm{AOR}=4.89$, $95 \% \mathrm{CI}=1.66-14.40)$, hypercholesterolemia $(\mathrm{AOR}=10.53,95 \% \mathrm{CI}=3.85-28.79)$ and presence of hypertension $(\mathrm{AOR}=3.76,95 \% \mathrm{CI}=1.62-8.72)$ were independent determinants of type 2 diabetes. In under- 


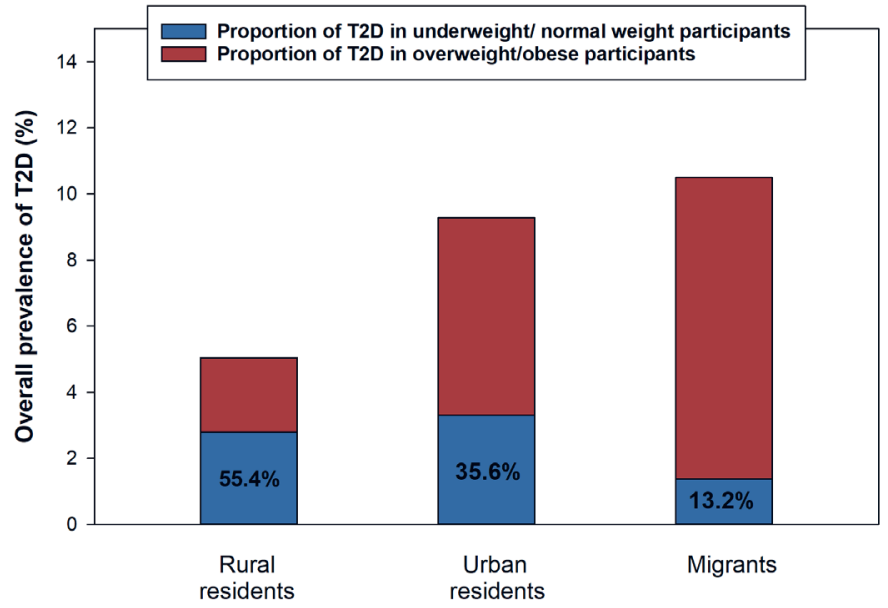

Figure 2. Proportion of type 2 diabetes in underweight / normal weight participants within the RODAM study. T2D - type 2 diabetes mellitus.

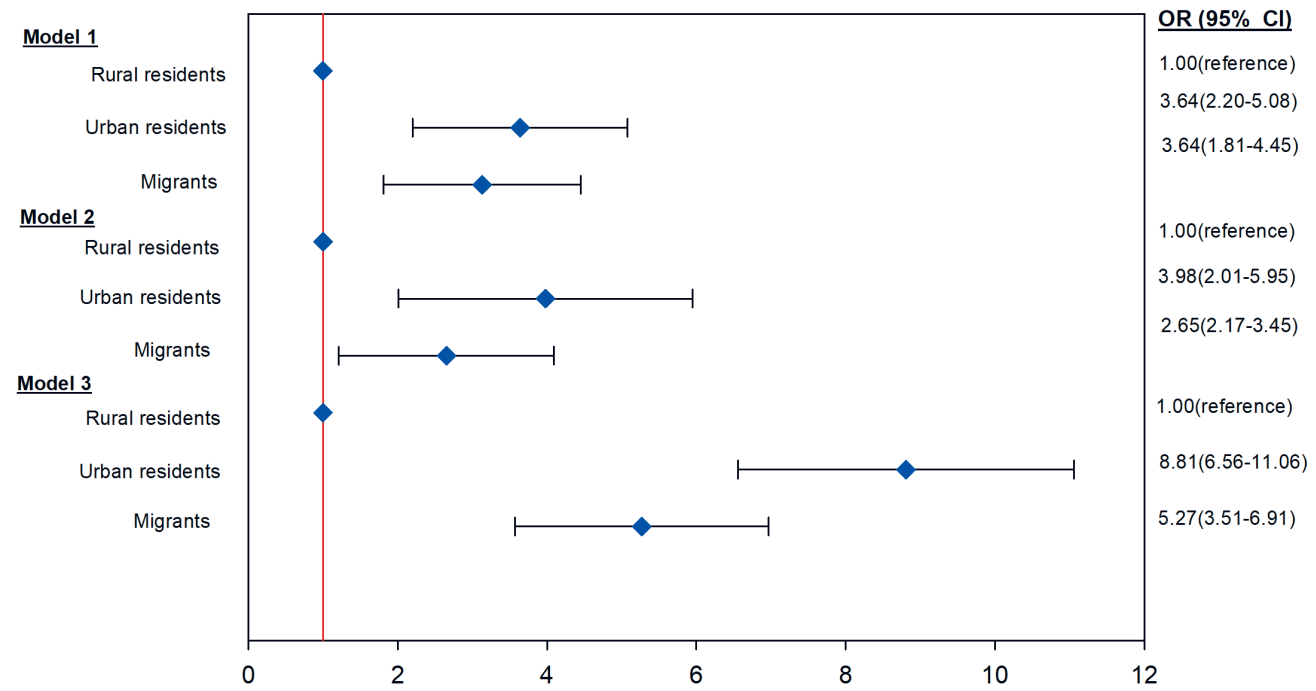

Figure 3. Odds of type 2 diabetes in underweight/normal weight urban residents and migrants, compared to rural - Ghanaians. Data are odds ratio (95\% Confidence Interval). Model 1 - adjusted for age, sex and education. Model 2 - Model 1 with further adjustment for alcohol consumption, smoking, physical activity, hypercholesterolemia, hypertension and length of stay in the migrants. Model 3 - Model 2 with further adjustment for blood glucose control (HbAlc $<7 \%$ ( $53 \mathrm{mmol} / \mathrm{mol}$ )) and diabetes treatment (diet, oral medication and insulin use).

weight/normal weight migrants, presence of hypertension ( $\mathrm{AOR}=4.45,95 \% \mathrm{CI}=1.38-14.32$ ) and older age ( $>65$ years) $(\mathrm{AOR}=9.84,95 \% \mathrm{CI}=2.22-23.71)$ were independently associated with type 2 diabetes (Table 2).

\section{DISCUSSION}

\section{Key findings}

Our study shows that type 2 diabetes in underweight/normal weight individuals is very common in different Ghanaian contexts. The highest prevalence of type 2 diabetes in all underweight/normal weight individuals is in urban residents. However, underweight/normal weight individuals with type 2 diabetes 


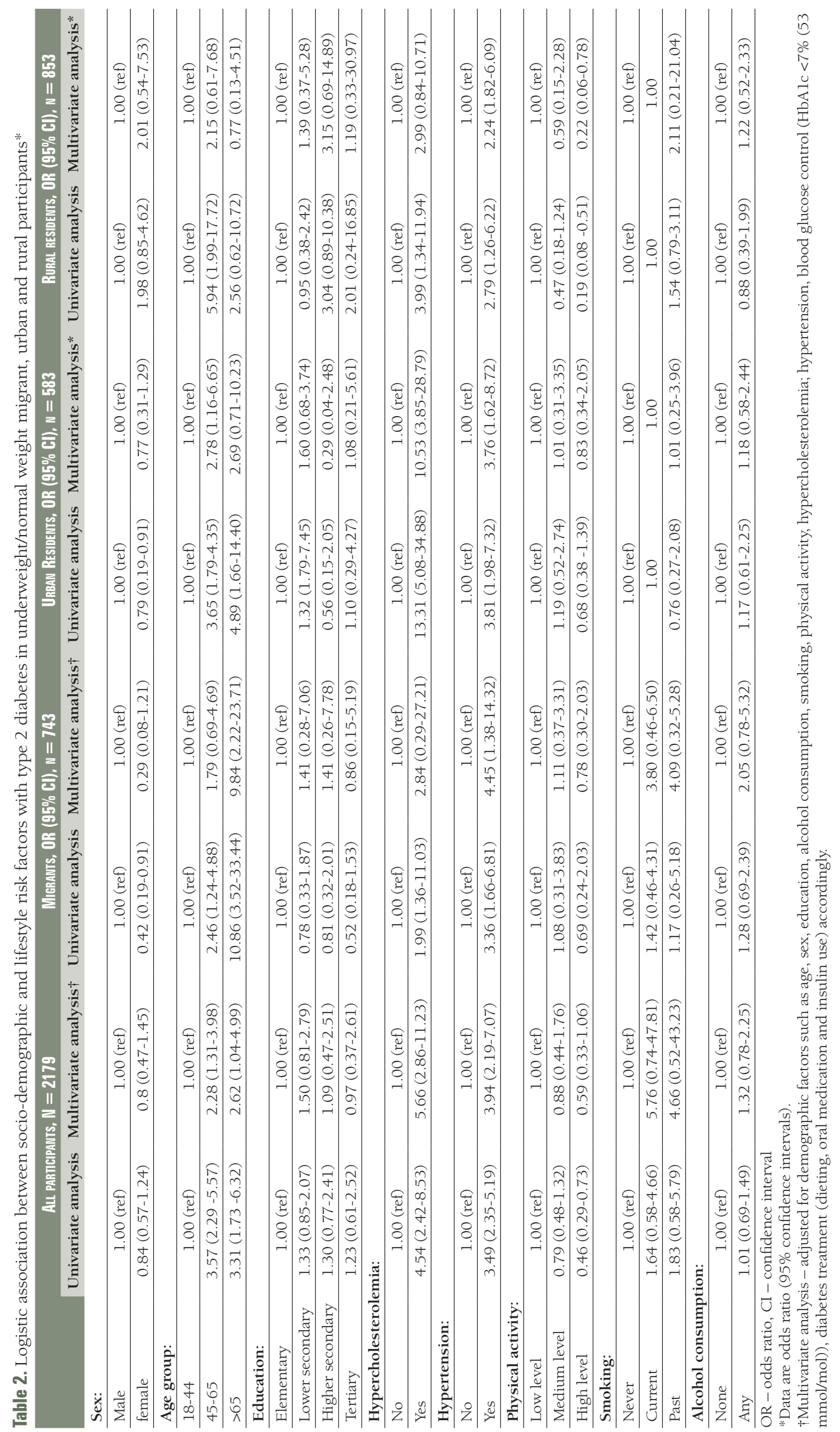


make up the highest proportion of all type 2 diabetes cases in rural residents. Determinants of type 2 diabetes in underweight/normal weight individuals include; presence of hypertension and physical inactivity among rural residents, middle age (45-65 years), hypercholesterolemia and presence of hypertension among urban residents, older age ( $>65$ years) and presence of hypertension among migrants.

\section{Interpretation of key findings}

In our study, we found that the overall prevalence of type 2 diabetes in underweight/normal weight individuals was $5.9 \%$, which is lower than the prevalence of type 2 diabetes in the overweight (10.3\%) and obese (12.2\%) reported from the same RODAM study [13]. This finding was expected since obesity is a well-known risk factor for type 2 diabetes, with the prevalence of type 2 diabetes increasing with increasing BMI [17]. In addition, our study found that the overall proportion of type 2 diabetes in underweight/ normal weight individuals with respect to all type 2 diabetes cases was 24\%, a figure which is higher than those reported in European or USA cohorts ie, $7.5 \%$ in the German DiaRegis (Diabetes treatment patterns and goal achievement in primary diabetes care) cohort, $8.4 \%$ in the German DIVE (Diabetes Versorgungs-Evaluation) cohort, 12\% in the NHS (Nurses' Health Study) and 16.5\% in HPFS (Health Professionals Follow-Up Study) cohort [18-20]. This finding is expected considering that risk factors for type 2 diabetes in underweight/normal weight individuals such as maternal undernutrition, low birth weight, childhood undernutrition are more common in SSA populations, hence predisposing SSA populations (including international migrants living in Europe) to type 2 diabetes as opposed to their European host populations $[11,21]$.

Our study is the first to mirror the epidemiological transition in Africa and report the differences in type 2 diabetes in underweight/normal weight individuals by location of residency. The highest odds of type 2 diabetes in underweight/normal weight individuals were higher in urban residents, followed by migrants. This finding was unexpected considering that fetal exposure to maternal undernutrition, pre-natal injurious agents and early childhood undernutrition are more common in the rural areas and could greatly increase the risk of type 2 diabetes in the rural group [16]. However, it should be noted that type 2 diabetes in underweight/normal weight rural residents accounted for the majority (55\%) of all type 2 diabetes cases, with only $13 \%$ of all type 2 diabetes cases in migrants consisting of underweight/ normal weight individuals. In our study, differences in the measured risk factors did not explain the observed higher odds of type 2 diabetes in urban residents compared to migrants. Previous studies have shown that metabolically unhealthy normal weight (MUNW) individuals are at risk of type 2 diabetes than metabolically healthy normal weight (MHNW) individuals [7]. MUNW encompasses glucose elevation in the presence of impaired insulin secretion, hypercholesterolemia, low leg fat mass, visceral obesity and fatty liver [7]. It is likely that the unmeasured MUNW contributes to the higher odds of type 2 diabetes in urban residents compared to migrants, but this need to be explored with further studies.

Variation of type 2 diabetes determinants by geographical context in underweight/normal weight individuals was anticipated. First, previous studies have reported that underweight/normal weight individuals at risk of type 2 diabetes present with a lipodystrophy like syndrome [7]. Evidence from our study showed that underweight/normal weight urban residents had a greater proportion of hypercholesterolemia compared to other groups, which would increase the risk of type 2 diabetes in this group. However, hypercholesterolemia results from a complex interplay of diet, physical activity and genetics. The higher levels of hypercholesterolemia in the urban residents could also be a direct result of physical inactivity and unhealthy diets as observed in our study [22].

Second, there is substantial overlap between diabetes and hypertension, reflecting a significant overlap in their etiology and disease mechanisms, including the sympathetic nervous system, renin-angiotensin-aldosterone system (RAAS), oxidative stress, adipokines, and peroxisome proliferator-activated receptors (PPARs) [23]. Hypertension has been reported in about $50 \%$ to $80 \%$ of individuals with type 2 diabetes [24]. It has also been reported that hypertensive subjects are 2.5 times more likely to develop type 2 diabetes [25]. In our study, hypertension was a determinant of type 2 diabetes in underweight/normal weight individuals in all three study groups. However, the odds of type 2 diabetes from hypertension were highest in underweight/normal weight individual migrants who also had the highest levels of hypertension at baseline compared to other groups.

It is worth noting that, individuals diagnosed with type 2 diabetes at older ages (60 years in men and 70 years in women in SSA) tend to be less overweight due to loss of muscle mass, impairment of pancreat- 
ic function, and increased intra-abdominal fat [26,27]. Therefore, it was not surprising that old age was associated with type 2 diabetes in underweight/normal weight individuals, especially in migrants. Nevertheless, the effects of age on type 2 diabetes were not apparent in underweight/normal weight rural residents, even though this group had the highest number of old participants. Thus, it is unclear why old age is not associated with type 2 diabetes in the underweight/normal weight rural group.

It is well established that physical exercise is protective against type 2 diabetes [28]. In our study, underweight/normal weight individuals with type 2 diabetes were 50\% less likely to have higher levels of physical activity compared to those without type 2 diabetes. High levels of physical activity were seen in rural residents, which may contribute to the lowest risk of type 2 diabetes in this group.

Alcohol consumption and smoking were not associated with type 2 diabetes in underweight/normal weight individuals. In contrast, previous studies have shown that alcohol consumption and smoking predispose individuals to type 2 diabetes in underweight/normal weight individuals, especially in the western context [7]. The lack of a significant association between alcohol consumption and smoking with type 2 diabetes in underweight/normal weight individuals in the current study may be due to the low prevalence of smoking and alcohol consumption among our study population. For example, the prevalence of smoking and high alcohol consumption were around $44.6 \%$ and $12 \%$ in the German DIVE study respectively, [18] whereas only $4.3 \%$ our respondents were smokers and $6.2 \%$ had any consumption of alcohol. It could be that our study did not have sufficient power to detect such associations.

The pathogenic mechanism of type 2 diabetes in underweight/normal weight individuals populations has been reported as predominantly beta cell failure, as opposed to insulin resistance, which is the major pathophysiology of type 2 diabetes in overweight/obese individuals [10]. In the subset of participants with IFG, we found higher odds of HOMA-B compared to HOMA-IR per increase in each SD. The attributable risk for HOMA-B was also higher than that of HOMA-IR. This finding shows that pancreatic beta cell failure also makes a relatively larger contribution to the pathophysiology of type 2 diabetes in underweight/normal weight SSA populations as opposed to insulin resistance [29].

\section{Strengths and limitations}

The strength of our study is that it assesses a homogenous population of migrant and non-migrant Ghanaians living in different settings in Africa and Europe using standardised methods, which mitigates the effects the effects of studying different SSA populations with dissimilar genetic background. Several limitations of this study should be considered. First, our data are cross-sectional and rely on self-reported measures for socio-demographic and lifestyle risk factors hence we cannot preclude the possibility of recall bias. Second, some participants were underweight/normal weight due to weight loss effects of type 2 diabetes medication (biguanides, GLP-1 receptor agonists, SLGT2 inhibitors), or even poor control of blood glucose [30-32]. We adequately controlled for these effects in the logistic regression models, thereby increasing the accuracy of our results. Third, we did not have data on early life adverse factors such as maternal undernutrition, low birthweight, prematurity, childhood malnutrition, epigenetics and measures of MUNW. Lastly, our numbers were too few to stratify the analysis by sex, hence we could not reported sex differences in our study.

\section{CONCLUSIONS}

Our findings are highly relevant to SSA countries with a predominantly rural population and undergoing rapid urbanization, yet have limited resources to tackle type 2 diabetes. Underweight/normal weight individuals are neglected in the prevention of type 2 diabetes since current recommendations for screening and lifestyle modifications are advised at a BMI greater than $25 \mathrm{~kg} / \mathrm{m}^{2}$. Worse still, rural areas have limited capacity to manage the detrimental effects of type 2 diabetes due to constrained resources. From our results, it is apparent that future studies should examine the pathophysiology of type 2 diabetes in underweight/normal weight individuals, including associations with maternal undernutrition, low birth weight, prematurity, childhood malnutrition, epigenetics, and MUNW concept. Furthermore, type 2 diabetes control efforts in SSA and African populations in Europe should be extended to underweight/normal weight individuals taking into account differences in rural, urban and migrant contexts. 


Acknowledgements: The authors are very grateful to the participants, research assistants, interviewers and
other staff of the five research locations who have taken part in the study.
Funding: This work was supported by the European Commission under the Framework Programme [Grant
Number: 278901$]$. FPC is supported by the Erasmus Mundus Joint Doctorate Program of the European Union
through the Amsterdam Institute of Global Health and Development (AIGHD) [Grant Agreement 2015-1595].
Authors contributions: FPC, PH, and CA conceived and designed the study. FPC analyses the data. FPC, wrote
the paper with the support of PH and CA. All authors FPC, PH, ARM, EB, KM, LS, JA, SB, ID, MBS, JS, EOD,
KKG, MMAMM and CA participated in drafting the article or revising it critically for content.
Competing interests: The authors completed the Unified Competing Interest form at www.icmje.org/coi_dis-
closure.pdf (available upon request from the corresponding author), and declare no conflicts of interest.
Additional material
Online Supplementary Document

1 Atun R, Davies JI, Gale EA, Bärnighausen T, Beran D, Kengne AP, et al. Diabetes in sub-Saharan Africa: from clinical care to health policy. Lancet Diabetes Endocrinol. 2017;5:622-67. Medline:28688818 doi:10.1016/S2213-8587(17)30181-X

2 Jamison DT, Alwan A, Mock CN, Nugent R, Watkins D, Adeyi O, et al. Universal health coverage and intersectoral action for health: key messages from Disease Control Priorities. Lancet. 2018;391:1108-20. Medline:29179954 doi:10.1016/ S0140-6736(17)32906-9

3 Chiu M, Austin PC, Manuel DG, Shah BR, Tu JV. Deriving ethnic-specific BMI cutoff points for assessing diabetes risk. Diabetes Care. 2011;34:1741-8. Medline:21680722 doi:10.2337/dc10-2300

4 Mokdad AH, Ford ES, Bowman BA, Dietz WH, Vinicor F, Bales VS, et al. Prevalence of obesity, diabetes, and obesity-related health risk factors, 2001. JAMA. 2003;289:76-9. Medline:12503980 doi:10.1001/jama.289.1.76

5 Gujral UP, Mohan V, Pradeepa R, Deepa M, Anjana RM, Narayan K. Ethnic differences in the prevalence of diabetes in underweight and normal weight individuals: The CARRS and NHANES studies. Diabetes Res Clin Pract. 2018;146:3440. Medline:30244052 doi:10.1016/j.diabres.2018.09.011

6 Kramer CK, Zinman B, Retnakaran R. Are metabolically healthy overweight and obesity benign conditions?: A systematic review and meta-analysis. Ann Intern Med. 2013;159:758-69. Medline:24297192 doi:10.7326/0003-4819-15911-201312030-00008

7 Stefan N, Schick F, Häring H-U. Causes, characteristics, and consequences of metabolically unhealthy normal weight in humans. Cell Metab. 2017;26:292-300. Medline:28768170 doi:10.1016/j.cmet.2017.07.008

8 Murphy N, Cross AJ, Abubakar M, Jenab M, Aleksandrova K, Boutron-Ruault M-C, et al. A nested case-control study of metabolically defined body size phenotypes and risk of colorectal cancer in the European Prospective Investigation into Cancer and Nutrition (EPIC). PLoS Med. 2016;13:e1001988. Medline:27046222 doi:10.1371/journal.pmed.1001988

9 Feeley A, Musenge E, Pettifor JM, Norris SA. Changes in dietary habits and eating practices in adolescents living in urban South Africa: The birth to twenty cohort. Nutrition. 2012;28:e1-6. Medline:22465902 doi:10.1016/j.nut.2011.11.025

10 George AM, Jacob AG, Fogelfeld L. Lean diabetes mellitus: An emerging entity in the era of obesity. World J Diabetes. 2015;6:613. Medline:25987958 doi:10.4239/wjd.v6.i4.613

11 Akombi BJ, Agho KE, Hall JJ, Wali N, Renzaho A, Merom D. Stunting, wasting and underweight in sub-Saharan Africa: a systematic review. Int J Environ Res Public Health. 2017;14:863. Medline:28788108 doi:10.3390/ijerph14080863

12 Davies JI, Macnab AJ, Byass P, Norris SA, Nyirenda M, Singhal A, et al. Developmental origins of health and disease in Africa—influencing early life. Lancet Glob Health. 2018;6:e244-5. Medline:29433658 doi:10.1016/S2214-109X(18)30036-6

13 Agyemang C, Beune E, Meeks K, Owusu-Dabo E, Agyei-Baffour P, Aikins AG, et al. Ad-G, et al. Rationale and cross-sectional study design of the Research on Obesity and type 2 Diabetes among African Migrants: the RODAM study. BMJ Open. 2014:4:e004877. Medline:24657884 doi:10.1136/bmjopen-2014-004877

14 Organization WH. Global database on body mass index: an interactive surveillance tool for monitoring nutrition transition. Public Health Nutr. 2006;9:658-60.

15 Bull FC, Maslin TS, Armstrong T. Global physical activity questionnaire (GPAQ): nine country reliability and validity study. J Phys Act Health. 2009;6:790-804. Medline:20101923 doi:10.1123/jpah.6.6.790

16 Wallace TM, Levy JC, Matthews DR. Use and abuse of HOMA modeling. Diabetes Care. 2004;27:1487-95. Medline:15161807 doi:10.2337/diacare.27.6.1487

17 Riobó Serván P. Obesidad y diabetes. Nutr Hosp. 2013;28:138-43. Medline:24010754

18 Hartmann B, Lanzinger S, Bramlage P, Groß F, Danne T, Wagner S, et al. Lean diabetes in middle-aged adults: A joint analysis of the German DIVE and DPV registries. PLoS One. 2017;12:e0183235. Medline:28827839 doi:10.1371/journal.pone.0183235

19 Hartmann B, Bramlage P, Schneider S, Tschöpe D, Gitt A. Impact of body weight on antidiabetic treatment and predictors of weight control under real-world conditions: a 2-year follow-up of DiaRegis cohort. Acta Diabetol. 2015;52:1093101. Medline:26239142 doi:10.1007/s00592-015-0794-0 
20 Tobias DK, Pan A, Jackson CL, O'reilly EJ, Ding EL, Willett WC, et al. Body-mass index and mortality among adults with incident type 2 diabetes. N Engl J Med. 2014;370:233-44. Medline:24428469 doi:10.1056/NEJMoal304501

21 Hanson MA, Gluckman P. Early developmental conditioning of later health and disease: physiology or pathophysiology? Physiol Rev. 2014;94:1027-76. Medline:25287859 doi:10.1152/physrev.00029.2013

22 Galbete C, Nicolaou M, Meeks K, Klipstein-Grobusch K, Aikins AG, Ado J, et al. Aikins Ad-G, Addo J, et al. Dietary patterns and type 2 diabetes among Ghanaian migrants in Europe and their compatriots in Ghana: the RODAM study. Nutr Diabetes. 2018;8:25. Medline:29695705 doi:10.1038/s41387-018-0029-x

23 Cheung BM, Li C. Diabetes and hypertension: is there a common metabolic pathway? Curr Atheroscler Rep. 2012;14:1606. Medline:22281657 doi:10.1007/s11883-012-0227-2

24 Landsberg L, Molitch M. Diabetes and hypertension: pathogenesis, prevention and treatment. Clin Exp Hypertens. 2004;26:621-8. Medline:15702616 doi:10.1081/CEH-200031945

25 Gress TW, Nieto FJ, Shahar E, Wofford MR, Brancati FL. Hypertension and antihypertensive therapy as risk factors for type 2 diabetes mellitus. N Engl J Med. 2000;342:905-12. Medline:10738048 doi:10.1056/NEJM200003303421301

26 Price AJ, Crampin AC, Amberbir A, Kayuni-Chihana N, Musicha C, Tafatatha T, et al. Prevalence of obesity, hypertension, and diabetes, and cascade of care in sub-Saharan Africa: a cross-sectional, population-based study in rural and urban Malawi. Lancet Diabetes Endocrinol. 2018;6:208-22. Medline:29371076 doi:10.1016/S2213-8587(17)30432-1

27 Goodpaster BH, Krishnaswami S, Resnick H, Kelley DE, Haggerty C, Harris TB, et al. Association between regional adipose tissue distribution and both type 2 diabetes and impaired glucose tolerance in elderly men and women. Diabetes Care. 2003;26:372-9. Medline:12547865 doi:10.2337/diacare.26.2.372

28 Lumb A. Diabetes and exercise. Clin Med (Lond). 2014;14:673-6. Medline:25468857 doi:10.7861/clinmedicine.14-6-673

29 Meeks KA, Stronks K, Adeyemo A, Addo J, Bahendeka S, Beune E, et al. Peripheral insulin resistance rather than beta cell dysfunction accounts for geographical differences in impaired fasting blood glucose among sub-Saharan African individuals: findings from the RODAM study. Diabetologia. 2017;60:854-64. Medline:28144712 doi:10.1007/s00125017-4216-4

30 Lau DC, Teoh H. Impact of current and emerging glucose-lowering drugs on body weight in type 2 diabetes. Can J Diabetes. 2015;39:S148-54. Medline:26654858 doi:10.1016/j.jcjd.2015.09.090

31 Grams J, Garvey WT. Weight loss and the prevention and treatment of type 2 diabetes using lifestyle therapy, pharmacotherapy, and bariatric surgery: mechanisms of action. Curr Obes Rep. 2015;4:287-302. Medline:26627223 doi:10.1007/ s13679-015-0155-x

32 Kalyani RR, Tra Y, Egan J, Ferrucci L, Brancati F. Hyperglycemia is associated with relatively lower lean body mass in older adults. J Nutr Health Aging. 2014;18:737-43. Medline:25286453 doi:10.1007/s12603-014-0538-9 